\title{
Perfuração de Ventrículo Esquerdo Durante Cateterismo Cardíaco Diagnóstico
}

\author{
Elias J. P. Conti ${ }^{1}$, Fábio M. Mota ${ }^{1}$, Leonardo G. Zanatta ${ }^{1}$, Marco T. Zanettini ${ }^{1}$, Alexandre S. Quadros ${ }^{1}$, \\ Rogério Sarmento-Leite', Carlos A. M. Gottschall ${ }^{1}$
}

\section{RESUMO}

Perfuração de uma câmara cardíaca é uma complicação maior do cateterismo cardíaco, condição extremamente rara, porém de gravidade bem documentada. Relatamos um caso de perfuração de ventrículo esquerdo ocorrido após a realização de ventriculografia esquerda com cateter pig tail, seguido de colapso cardiovascular em decorrência de tamponamento cardíaco. O quadro foi manejado com pronta realização de pericardiocentese, a qual foi suficiente para estabilização clínica e resolução do caso.

DESCRITORES: Cateterismo cardíaco/efeitos adversos. Cineangiografia/efeitos adversos. Ventrículos do coração. Tamponamento cardíaco/etiologia. Traumatismos cardíacos/etiologia.
D urante o cateterismo cardíaco diagnóstico, a perfuração cardíaca é de ocorrência extremamente rara, mas de potencial gravidade. Em decorrência da crescente indicação e realização de procedimentos coronários invasivos, as complicações têm se tornado mais freqüentes quando se consideram números absolutos, apesar da maior experiência dos operadores e do aperfeiçoamento do material e da técnica ${ }^{1-4}$. Existem poucos relatos disponíveis de ruptura de ventrículo esquerdo conseqüente à realização da ventriculografia, dando maior ênfase à raridade dessa complicação $0^{5,6}$.

\section{RELATO DO CASO}

Paciente de 72 anos, sexo feminino, branca, foi encaminhada para realização de cinecoronariografia eletiva por suspeita clínica de cardiopatia isquêmica.

\footnotetext{
1 Instituto de Cardiologia do Rio Grande do Sul/Fundação Universitária de Cardiologia - Porto Alegre, RS.

Correspondência: Rogério Sarmento-Leite. Av. Princesa Isabel, 370

- Santana - Porto Alegre, RS - CEP 90620-000

E-mail: sleite.pesquisa@cardiologia.org.br

Recebido em: 30/6/2008 • Aceito em: 22/8/2008
}

\begin{abstract}
SUMMARY
Perforation of the Left Ventricle During Cardiac Catheterization

Perforation of a heart chamber is a major complication of cardiac catheterization, an extremely rare condition, although its potential severity is well documented. We describe a case of perforation of the left ventricle occurred after a left ventriculography with a pig tail catheter, followed by cardiovascular collapse due to cardiac tamponade. This situation was handled with prompt pericardiocentesis, which led to clinical stabilization and case resolution.
\end{abstract}

DESCRIPTORS: Heart catheterization/adverse effects. Cineangiography/adverse effects. Heart ventricles. Cardiac tamponade/etiology. Heart injuries/etiology.

Eletrocardiograma de repouso revelou ritmo sinusal, sinais de sobrecarga ventricular esquerda e alterações mistas da repolarização ventricular. A paciente apresentava como co-morbidades hipertensão arterial sistêmica, em tratamento com atenolol e hidroclorotiazida, e hipotireoidismo, em uso de tiroxina $100 \mu \mathrm{g} / \mathrm{dia}$. Não havia história prévia de diabetes, dislipidemia, tabagismo, alergias ou distúrbios da coagulação.

A abordagem para cinecoronariografia foi pela via femoral direita, utilizando introdutor 6F (french). A guia 0.035" cruzou a valva aórtica sem resistência, e o cateter pig tail foi deslizado sobre ela sem dificuldade, até o ápice do ventrículo esquerdo. Após lavagem do cateter e adequado posicionamento do mesmo, conferiu-se a forma de onda de pressão do ventrículo esquerdo e, em seguida, foram injetados $30 \mathrm{ml}$ de contraste a $15 \mathrm{ml} / \mathrm{s}$ e 500 psi. Visualizou-se o ventrículo esquerdo hipertrófico com boa contratilidade e extravasamento de grande quantidade de contraste para fora do ventrículo esquerdo, através de seu ápice, distribuindo-se por todo o saco pericárdico (Figura 1). Alguns minutos após, a paciente apresentou instabilidade hemodinâmica, evoluindo para choque cardiogênico.

Considerou-se que a piora hemodinâmica foi secundária ao tamponamento cardíaco induzido por 
Conti EJP, et al. Perfuração de Ventrículo Esquerdo Durante Cateterismo Cardíaco Diagnóstico. Rev Bras Cardiol Invas. 2008;16(3):365-367.

perfuração do ventrículo esquerdo e procedeu-se à punção subxifóide seguida de imediata aspiração de $60 \mathrm{ml}$ de sangue com pronta melhora hemodinâmica. Após, foi introduzido guia de Amplatzer através da agulha de punção no saco pericárdico, e sobre a guia foi colocado cateter pig tail 5F no espaço pericárdico, mantido sob pressão negativa. Nos trinta minutos subseqüentes, foram drenados $80 \mathrm{ml}$ de sangue através do cateter, cessando o sangramento. Decidiu-se por prosseguir a cinecoronariografia, no intuito de descartar doença arterial coronária como causa de fragilidade ventricular esquerda, tendo sido demonstradas artérias coronárias normais (Figura 2). Ecocardiograma de controle após estabilização, ainda na sala de Hemodinâmica, evidenciou mínima lâmina de líquido intrapericárdico, sem outras particularidades.

A paciente foi transferida para Unidade de Terapia Intensiva (UTI), onde permaneceu estável, sendo realizado novo eletrocardiograma e nova radiografia de tórax (Figura 3), os quais não apresentaram alterações. Ecocar-

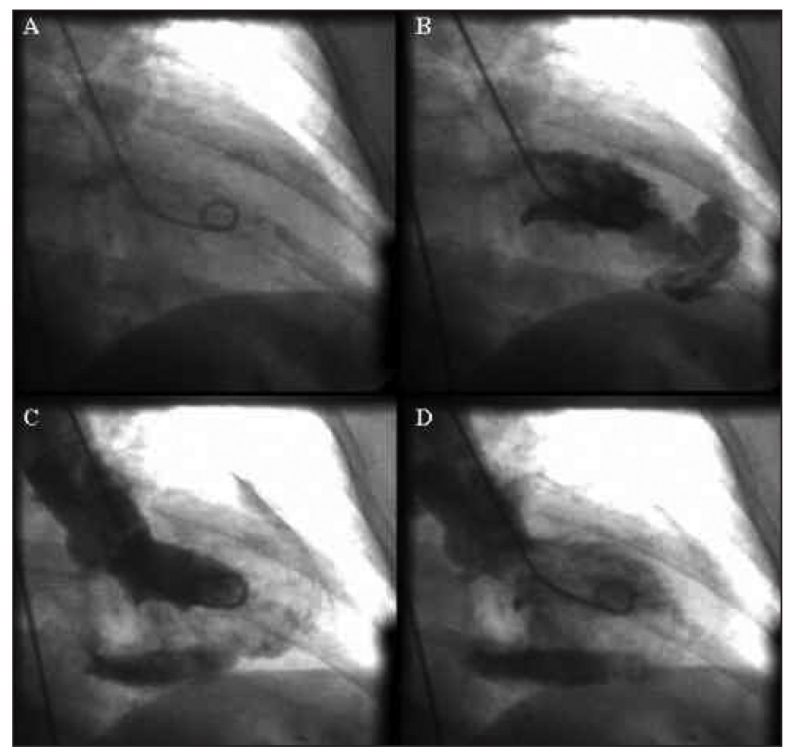

Figura 1 - Ventriculografia em projeção oblíqua anterior direita 30 graus, com extravasamento de contraste para o saco pericárdico.

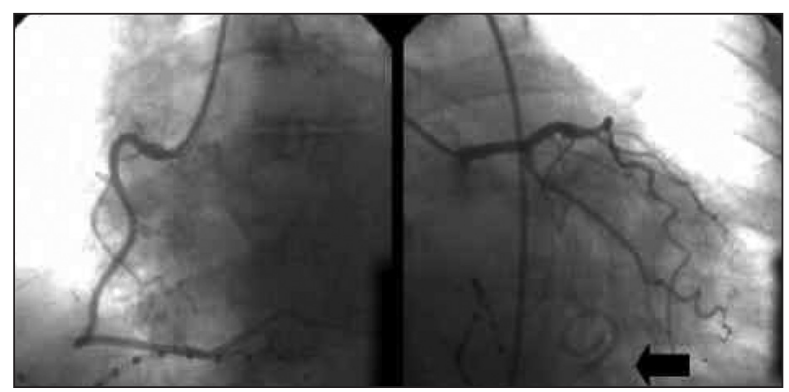

Figura 2 - Coronariografia demonstrando artérias coronárias sem lesões. No detalhe (seta), cateter pig tail posicionado no espaço pericárdico.

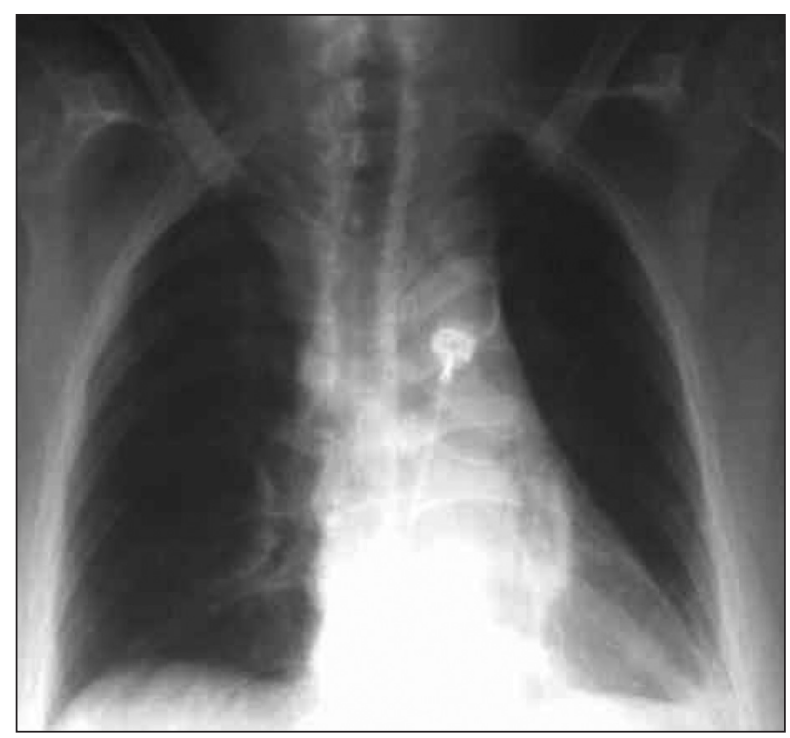

Figura 3 - Radiografia de tórax de controle, aproximadamente duas horas após o evento.

diograma de controle após seis horas do início do quadro mostrou-se semelhante ao prévio, com mínima lâmina de líquido intrapericárdico. Diante da estabilidade clínica, da ausência de drenagem e do resultado favorável da avaliação complementar, optou-se por retirar o cateter pig tail do saco pericárdico. A paciente teve boa evolução, recebendo alta hospitalar no quinto dia de internação. Em consulta ambulatorial três meses após, apresenta-se bem e sem queixas cardiovasculares.

\section{DISCUSSÃO}

A incidência de perfuração de uma câmara cardíaca é de 0,03\% em registro da SCAI (Society for Cardiovascular Angiography and Intervention), em que foram avaliados 59.792 pacientes submetidos a cateterismo cardíaco ${ }^{7}$. O risco é relacionado a diversas variáveis e está aumentado em procedimentos que demandam maior trauma, como biópsia endomiocárdica, cateterismo com punção transeptal, valvuloplastia com balão, colocação de marca-passo e pericardiocentese com agulha, além do uso de guias e cateteres mais rígidos do tipo stiffer ${ }^{8}$. Mulheres em idade avançada parecem apresentar maior risco, provavelmente pelo adelgaçamento das paredes, em especial de câmaras direitas ${ }^{8}$.

Pronto reconhecimento e rápido tratamento dessa condição são essenciais, já que se trata de uma complicação potencialmente fatal. Bradicardia associada à hipotensão grave que se desenvolve durante ou logo após o cateterismo deve suscitar o diagnóstico ${ }^{8}$. Em relação ao tratamento, a pericardiocentese via subxifóide guiada por ultra-sonografia é a abordagem de escolha nos casos em que se sucede colapso cardiovascular por tamponamento cardíaco ${ }^{4}$. Em estudo com 91 pacientes 

$2008 ; 16(3): 365-367$.

que desenvolveram tamponamento agudo durante o cateterismo, pericardiocentese foi a única terapia necessária em $82 \%$ dos $\operatorname{casos}^{4}$. O procedimento é o tratamento definitivo para a maioria dos pacientes, deixando-se os casos refratários, isto é, aqueles nos quais a drenagem não cessa mesmo após a reversão da anticoagulação, para tratamento cirúrgico (Figura 2).

Como medida preventiva em procedimentos invasivos, recomenda-se o adequado e cuidadoso posicionamento dos fios-guias e cateteres, procurando-se evitar, particularmente na cavidade ventricular, seu aprisionamento nas trabéculas ou nos músculos papilares, que, embora de forma infreqüente, pode ocasionar complicações potencialmente graves.

\section{REFERÊNCIAS BIBLIOGRÁFICAS}

1. Friedrich SP, Berman AD, Baim DS, Diver DJ. Myocardial perforation in the cardiac catheterization laboratory: incidence, presentation, diagnosis, and management. Cathet Cardiovasc Diagn. 1994;32(2):99-107.

2. Von Sohsten R, Kopistansky C, Cohen M, Kussmaul WG $3^{\text {rd }}$. Cardiac tamponade in the "new device" era: evaluation of 6999 consecutive percutaneous coronary interventions. Am Heart J. 2000;140(2):279-83.

3. Wyman RM, Safian RD, Portway V, Skillman JJ, McKay RG, Baim DS. Current complications of diagnostic and therapeutic cardiac catheterization. J Am Coll Cardiol. 1988;12(6):1400-6.

4. Tsang TS, Freeman WK, Barnes ME, Reeder GS, Packer DL, Seward JB. Rescue echocardiographically guided pericardiocentesis for cardiac perforation complicating catheterbased procedures. The Mayo Clinic experience. J Am Coll Cardiol. 1998;32(5):1345-50.

5. Levin AR, Spach MS, Anderson PA, Capp MP. Cardiac perforation following left ventricular cineangiocardiography. Circulation. 1965;32(4):593-6.

6. Turk T, Vural AH, Ata Y, Ozkan H, Yavuz S. Left ventricular perforation during cardiac catheterization: a case report. Turkish J Thorac Cardiovasc Surg. 2008;16(1):43-4.

7. Scanlon PJ, Faxon DP, Audet AM, Carabello B, Dehmer GJ, Eagle KA, et al. ACC/AHA guidelines for coronary angiography: A report of the American College of Cardiology/American Heart Association Task Force on Practice Guidelines (Committee on Coronary Angiography). J Am Coll Cardiol. 1999;33(6):1756-824.

8. Baim DS, Simon DI. Cardiac catheterization. In: Baim DS, Grossman W, eds. Angiography and intervention. $7^{\text {th }}$ ed. New York: Williams \& Wilkins; 2006. p.36-76. 